\title{
BI-MODAL PALM PRINT AND KNUCKLE PRINT RECOGNITION SYSTEM
}

\author{
Michael K.O. Goh ${ }^{1}$, Connie Tee ${ }^{1}$, Andrew B.J. Teoh ${ }^{2}$ \\ ${ }^{I}$ Faculty of Information Science \& Tech. \\ Multimedia University \\ Jalan Ayer Keroh Lama, Melaka \\ Malaysia \\ ${ }^{2}$ School of Electrical \& Electronic Engineering \\ Yonsei University, Seoul, \\ South Korea
}

\begin{abstract}
This paper proposed an innovative contact-less palm print and knuckle print recognition system. Palm print is referred to as line textures, which contains principal lines, wrinkles and ridges on the inner surface of the palm. On the other hand, knuckle print is denoted as the flexion lines on the inner skin of the knuckles of the fingers. These line patterns are unique and stable, and they offer abundance of useful information for personal recognition. We present a novel palm print and knuckle print tracking approach to automatically detect and capture these features from low resolution video stream. No constraint is imposed and the subject can place his/her hand naturally on top of the input sensor without touching any device. The palm print and knuckle print features are extracted using our proposed Wavelet Gabor Competitive Code and Ridget Transform methods. Several decision-level fusion rules are used to consolidate the scores output by the palm print and knuckle print experts. The fusion of these features yields promising result of $E E R=1.25 \%$ for verification rate.
\end{abstract}

Keywords: Palm print recognition, knuckle print recognition, real-time hand tracking, ridgelet transform, score level fusion.

\section{INTRODUCTION}

In recent years, many security systems yearn for a more reliable and practical biometric technology. Hand-based biometrics, such as fingerprint and hand geometry, is the most prevalent biometric system in the marketplace (Anonymous, 2003). However, fingerprint suffers from a major drawback, which is its proneness to antisecurity threats, such as the reproduction of fingerprints left on surfaces to deceive the system. On the other hand, the hand geometry features are not descriptive enough for identification when the number of users grows larger. In this paper, we explore an alternative way to utilize palm print and knuckle print features for biometric recognition. Palm print refers to the visible principal lines, wrinkles, and ridges on the palm's surface (Figure 1(a)), while knuckle print denotes the flexion shrinks in the inner skin of knuckles (Figure 1(b)). Similar to fingerprint, these dermal patterns are formed at birth and they will not change throughout the life of a person. These line features are reliable and they can serve as unique personal identifier. Moreover, these line textures are clearly visible on the hand's surface and they can be captured using relatively inexpensive low-resolution device.

Researchers have proposed several promising methods for palm print biometrics. The works reported in the literature can be broadly classified into three categories: line-based, subspace-based, and transform-based. The line-based approach studies the structural information of the palm print. Line patterns such as principle lines, wrinkles, ridges, and creases are extracted for recognition (Funada et al., 1998; Duta et al., 2002; Chen et al., 2001). The later researches used more flexible approach to extract the palm lines by using edge detection methods, for example Sobel operator (Wu et al., 2004; Boles \& Chu, 1997; Leung at al., 2007), morphological operator (Rafael Diaz et al., 2004), edge map (Kung et al., 1995), and modified radon transform (Huang et al., 2008). The subspace-based approach is more straightforward and it treats the palm print image as a whole. Common subspace methods include Principal Component Analysis (PCA) (Lu et al., 2003), Linear Discriminant Analysis (LDA) (Wu et al., 2003), and Independent Component Analysis (ICA) (Connie et al., 2005). The transform-based technique utilises some statistical approaches to transform the palm print images into another 
domain. The common methods used include Gabor (You et al., 2004), wavelets (Wu et al., 2002)), and Fourier transform ( $\mathrm{Li}$ et al., 2003). On the other hand, knuckle print is a relatively new biometric modality. Although it is intuitive to use knuckle print texture on the fingers for recognition, it is to our knowledge that only a few works study the knuckle print as a biometric characteristic. Li et al. (2003) used a hierarchical classification method to study knuckle print based on location and line features (Li et al., 2004). In (Ribaric \& Fratric, 2005), Principal Component Analysis was employed to project finger images into lower dimensional subspace. Apart from that, Loris et al. (2009) also investigated knuckle features by fusing the knuckle print pattern from the middle and ring fingers. Good experiment result had testified that knuckle print is a reliable biometric modality.

All of the studies described above are based on touch-based approach. The users need to touch the sensor in order for their hand images to be acquired. The touch-based approach introduces several problems. For example, the users might be concern with the sanitary issue when they place their hand on the same sensor where countless others have also placed theirs. This problem is particularly exacerbated in some countries at the height of SARS or H1N1 epidemic. Apart from that, the surface will get contaminated easily if not used correctly, especially in harsh, dirty, and outdoor environments. In addition, some conservative nations may resist placing their hands after a user of the opposite sex has touched the sensor.

This paper investigates the use of a contact-less palm print and knuckle print recognition system. A novel palm and knuckle print tracking algorithm is proposed to detect and extract the palm and knuckle prints features simultaneously for recognition. There are several motivations for fusing the palm print and knuckle print biometric systems. Apart from higher performance and spoof resilience, the advantages of the proposed bimodal system include:

- Two independent features originate from the same part of the body, namely the palm and knuckle prints, can be extracted for verification simultaneously (reduces redundancy).

- Users can be shielded completely from the complexity of multimodal verification system using a single sensor, as they do not need to go through the complication of undergoing multiple sensors for security verification. This increases the user-friendliness of the system.

- Cost of implementation and maintenance can be reduced as compared to other multimodal biometric systems which require multiple sensors.

The contributions of this paper are two-fold. Firstly, we propose a novel contact-less palm print and knuckle print recognition based on low-resolution video camera. The implementation and analysis of a real-time videobased hand tracking system is presented. The proposed hand tracking algorithm has fault tolerant ability for slight translations, rotations, and distortions. Secondly, we propose a bi-modal biometric system design based on palm print and knuckle print matchers. The use of the proposed combination strategy in combining the two matchers significantly improves the overall accuracy of the biometric system.

The subsequent sections of this paper are organised as follows. Section 2 describes the methods used for the proposed bi-modal palm print and knuckle print system. Right after are the details of the experimental setup of the proposed system provided in Section 3. Next, the experimental results are presented and discussed in Section 4 , and this is then followed by some concluding remarks in Section 5.

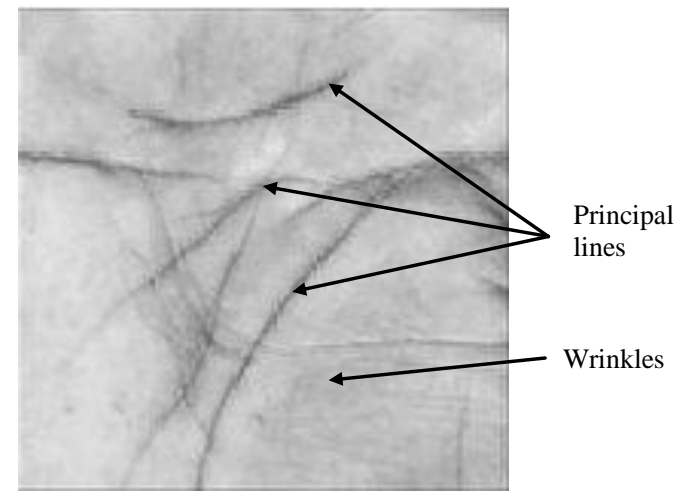

(a)

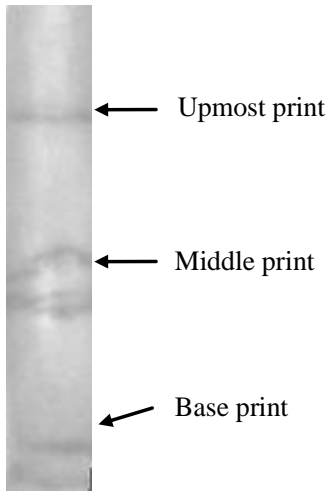

(b)

Figure 1: (a) Palm print. (b) Knuckle print. 


\section{PROPOSED WORK}

In this paper, we propose a bi-modal biometric system, which extracts the palm print and knuckle print images from real-time video stream for recognition. We utilised the knuckle print from four fingers, namely the pointed, middle, index and little fingers. The knuckle prints of interest refer to the horizontal-like lines distributed on the palm area of the knuckles. Due to regular bending exercise of the knuckles, these flexion lines are very obvious as compared to the surrounding skin area on the fingers. The knuckle prints usually appear on three locations in which we denote as upmost, middle, and base prints (refer to Figure 1(b)). Among the three locations, middle prints contribute the most features for recognition as it constitutes richer line texture. As for palm print, only the centre region of the palm area is employed as region of interest (ROI) and it contains important line patterns that are the three principal lines and dominant wrinkles. The following section discusses the automatic tracking algorithm to detect and to extract knuckle print and palm print for recognition.

\subsection{Automatic Tracking of Palm Print and Knuckle Print}

We propose a real-time hand tracking algorithm. This is a challenging issue since the hand exhibits significant amounts of articulation and self-occlusion that cause difficulties with existing algorithm. To further exasperate the problems, interactive biometric system requires that the hand tracking be performed in real-time.

We follow the Competitive Hand Valley Detection (CHVD) algorithm discussed in our previous paper (Michael et al., 2008) to locate the region of interest (ROI) of the palm. According to this algorithm, the ROI of the palm can be determined by the following landmark points on the hand contour: $p_{1}, p_{2}, p_{3}$, and $p_{4}$ (Figure 2). In this paper, we extended the CHVD algorithm to track the knuckle print image.

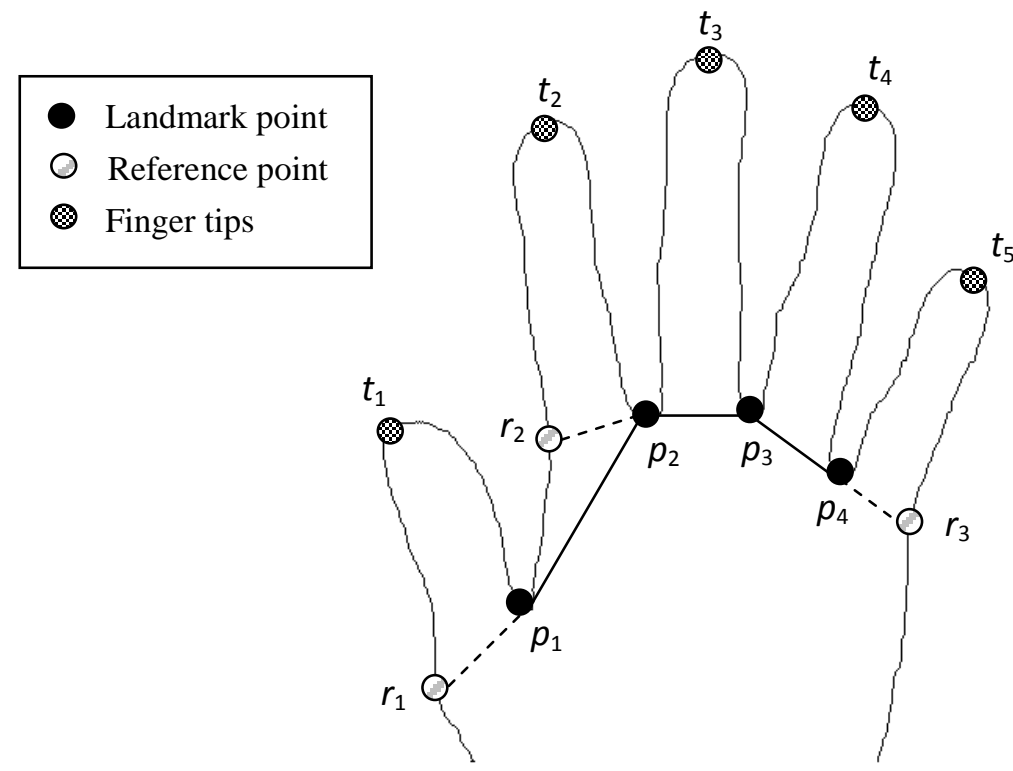

Figure 2: Locations of the four hand valley points and the three reference points.

After obtaining the four landmark points, denoted $\left\{p_{\mathrm{i}} \mid \mathrm{i}=1, \ldots, 4\right\}$, we find another three reference points, $\left\{r_{\mathrm{i}} \mid\right.$ $\mathrm{i}=1, \ldots, 3\}$, crucial for extracting the knuckle prints. These three points are located at the edges of the thumb, index and little fingers as shown in Figure 2. These reference points are situated at the zero-crossing points of the second derivation of the image profile and they can be found by extending a straight line from the landmark points to intersect the edge of the hand contour.

The next step is to locate the finger tips, denoted as $\left\{t_{\mathrm{i}} \mid \mathrm{i}=1, \ldots, 5\right\}$, by deploying a rather simple but effective method named projectile search approach. This algorithm gets its name from its upward search for the finger tips, following a trajectory-like path in the projectile motion. Instead of checking every contour pixel, we propel a point upwards from the base of the fingers. The point traverses within the vicinity of the finger until it reaches the tip. During its journey, the point may hit the boundary of the hand contour (wall) and "bounces" to the opposite wall to continue its journey to the tip. This algorithm greatly speeds up the search by omitting large number of contour point hunts. 
Consider the finger profile shown in Figure 3, the midpoint between the valleys of the fingers, $m_{i}$, was first determined using $\left(\frac{x_{p_{i}}+x_{p_{i+1}}}{2}, \frac{y_{p_{i}}+y_{p_{i+1}}}{2}\right)$. Next, we project a point from $m_{i}$ to touch the finger tip $t_{i}$ (Figure 3(a)). This point traverses in upwards direction until it reaches the zero-crossing point at the edge of the finger, where the finger tip is located. After that, a vertical line, $L_{i}$, is drawn to connect $m_{i}$ and $t_{i}$ (Figure 3(b)). A horizontal line, $L_{\perp_{i}}$, (Figure 3(c)) is then set with the equation $y_{L \perp_{i}}=y_{t_{i}}+0.1\left\|L_{i}\right\|$, where $\|\cdot\|$ denotes the magnitude of the line. In this experiment, $L_{\perp_{i}}$ must be made perpendicular to $L_{i}$ by fulfilling the constraint, $\nabla L \perp_{i}=-\frac{1}{\nabla L_{i}}$, where $\nabla$ refers to the line gradient. Similarly, another horizontal line, $L^{\prime}{ }_{\perp_{i}}$, near the base of the finger is determined using the equation $y_{L^{\prime} \perp_{i}}=y_{\overline{p_{i} p_{i+1}}}+0.05\left\|L_{i}\right\|$. Subsequently, a rectangular profile covering the area between the tip and base of the finger can be found (Figure 3(d)). Conditions are imposed to determine the way to form this rectangle,

$$
w_{t}=\left\{\begin{array}{ll}
w_{b} & \text { if } w_{b}<w_{t} \\
w_{t} & \text { otherwise }
\end{array} \text { and } w_{b}= \begin{cases}w_{t} & \text { if } w_{t}<w_{b} \\
w_{b} & \text { otherwise }\end{cases}\right.
$$

where $w_{t}$ signifies the width of the top line segment near the finger tip and $w_{b}$ represents the base line segment above the finger valley. Finally, the ROI of the knuckle print can be obtained by cropping this rectangular segment (Figure 3(e))

The algorithm discussed can only be applied to "ideal" cases where the fingers are in an upright position. In cases which fingers are oriented to an angle (Figure 3(f) and (k)), the same algorithm with slight variation must be used. Consider finger in Figure 3(f) (or Figure 3(k) in the opposite direction), a point is projected from the midpoint $m_{i}$. As the finger is displaced away from the vertical axis, the point cannot traverse directly to reach the tip. It hits the wall of the hand contour along its way and "bounces" to the opposite wall following a horizontal path. After reaching the opposite wall, the point will "reinitialise" its position and project upwards again from the midpoint of the horizontal path. The point continues its journey to the tip of the finger in a similar "zigzag" manner. After locating the tip of the finger, the same process as described previously can be applied to find the ROI of the knuckle print.

Figure 4 illustrates the ROIs of the knuckle print and palm print detection using the proposed algorithm. It works well for both left and right hands.

\subsection{Extracting Knuckle Print Feature}

Here, we adopted Ridgelet transform to extract the prominent knuckle print features. The method works specifically on images with lines. Since knuckle print is mainly made up of horizontal-like ridge lines, Ridgelet method would be able to deal with the line pattern more effectively as compared to other methods such as Wavelets. Discrete Ridgelet transform can also deal with line singularity effectively through Radon transform, and then using Wavelet transform on each projection in the Radon transform domain ( Candes \& Donoho, 1999; Donoho, 2000; Do and Vetterli, 2003). The idea behind Ridgelet transform was to map a line singularity to a point singularity by using Radon transform (Deans, 1983) and then using Wavelet transform to represent the point singularity in the Radon transform. Ridgelet transform represents straight edges in images using a few coefficients where the most significant coefficient shows the directions of the image lines with the highest energy.

In order to apply Ridgelets transform to digital images, Discrete transform is required. Do \& Vetterli (2003) proposed Digital form of Ridgelets transform called Finite Ridgelet Transform (FRIT) (Candes \& Donoho, 1999). FRIT is accomplished through the Finite Radon Transform (FRAT) which is defined as the summation of image pixel values along a set of lines. Consider $Z_{p}=\{0,1, \ldots, p-1\}$, where $p$ is a prime number, the FRAT of a real function, $f(x, y)$, on the finite $p \times p$ lattice $Z_{p}^{2}$ is defined as

$$
\operatorname{FRAT}[k, m]=\frac{1}{\sqrt{p}} \sum_{(x, y) \in L_{k, m}} f[x, y],
$$




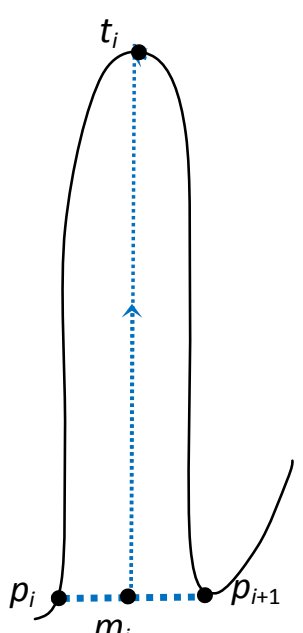

(a)

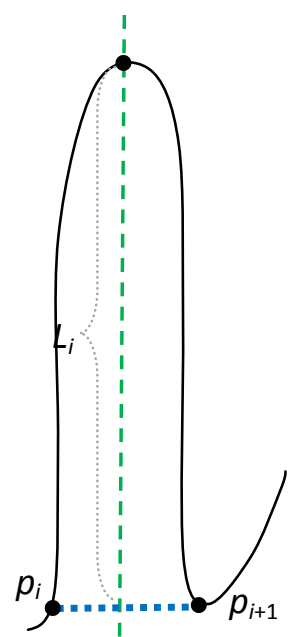

(b)

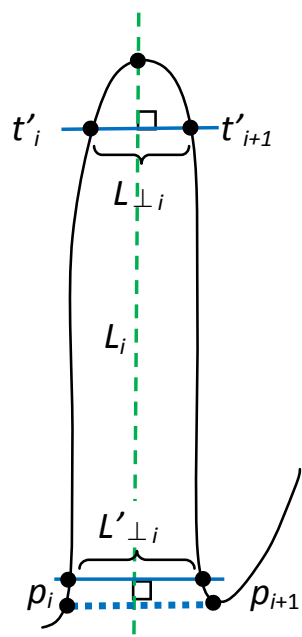

(c)

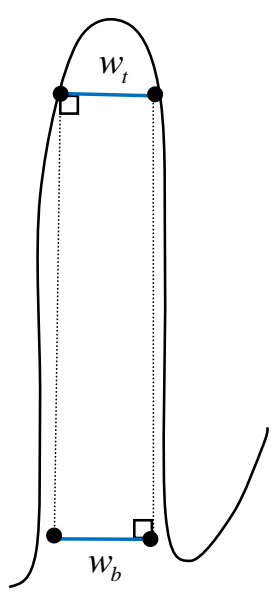

(d)

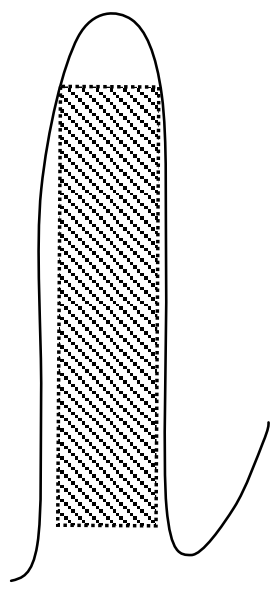

(e)
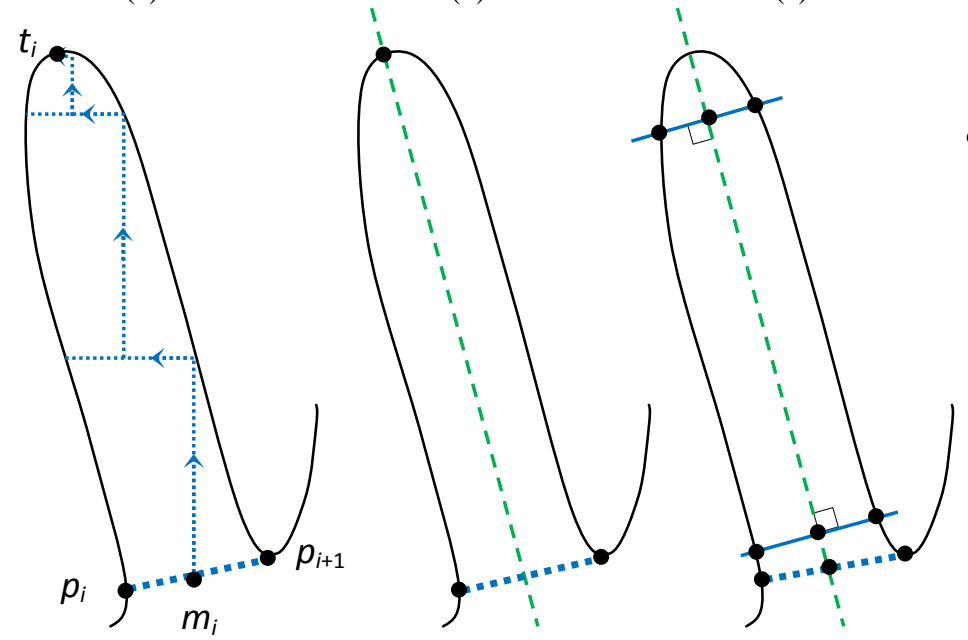

(h)

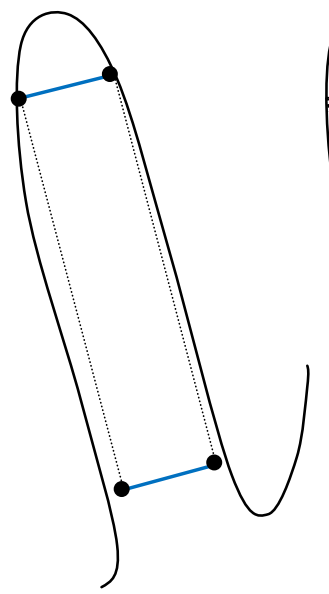

(i)

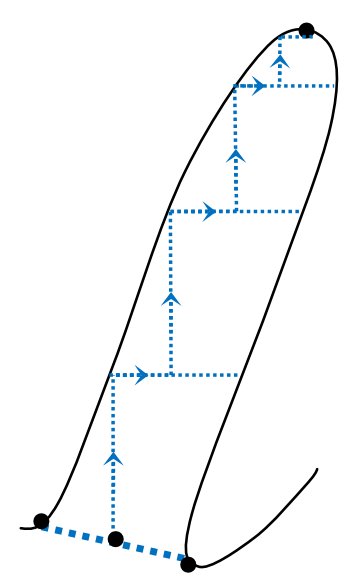

(k)

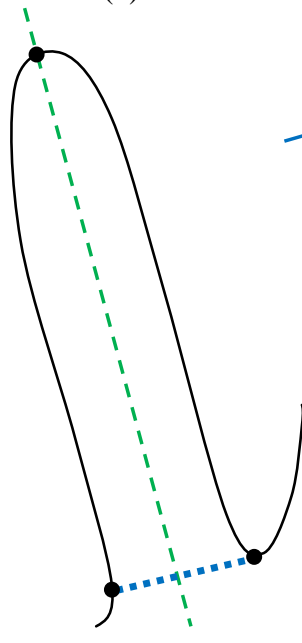

(g)

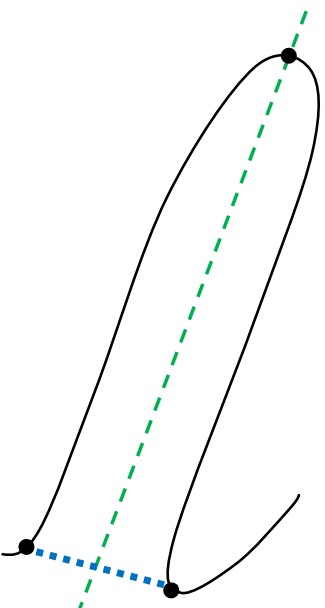

(1)

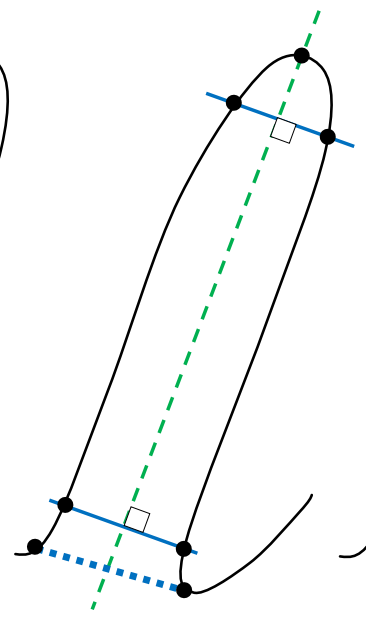

(m)

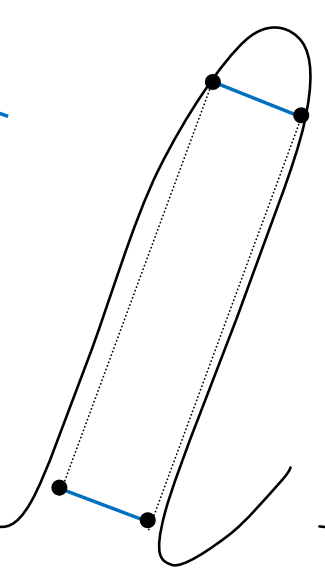

(n)

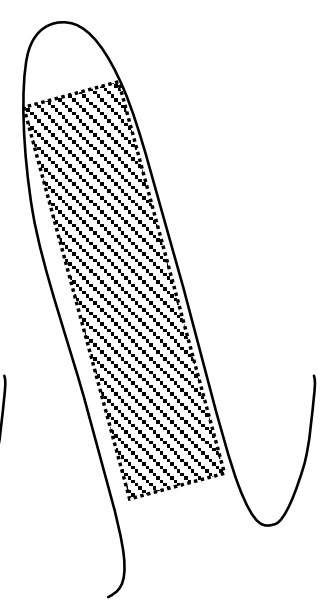

(j)

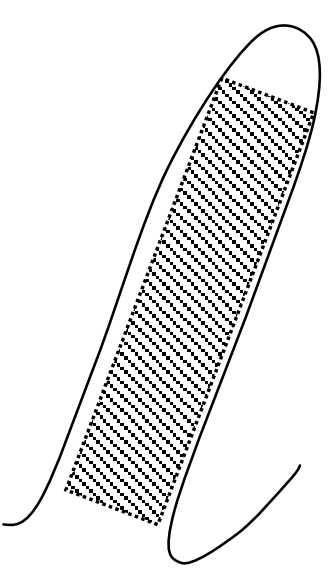

(o)

Figure 3: The illustration of the proposed projectile search algorithm for a finger. 

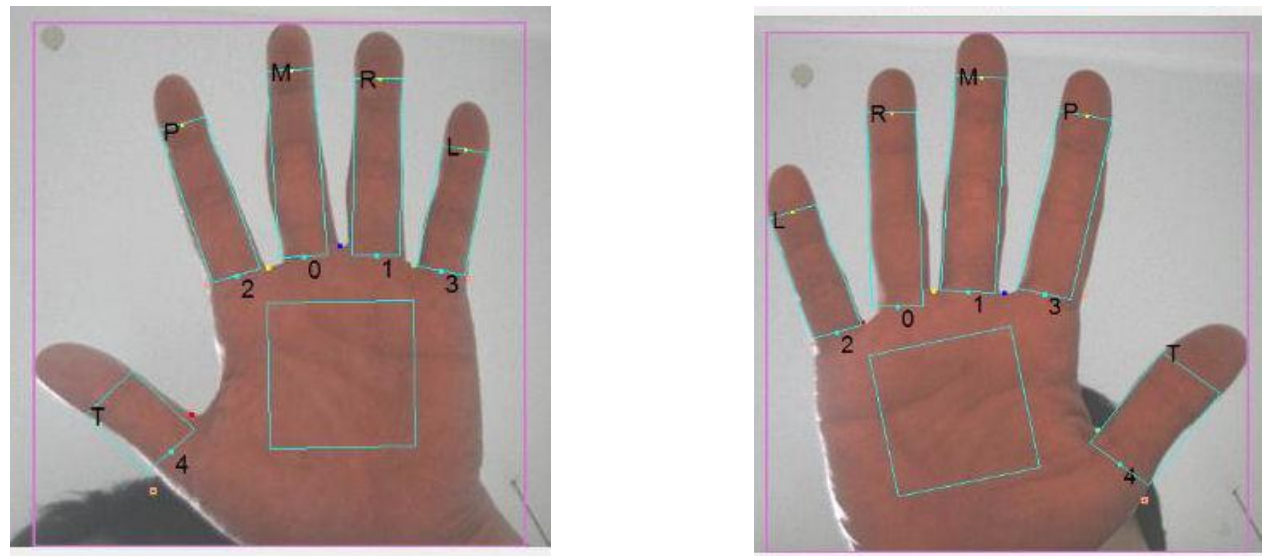

Figure 4: ROIs of palm print and knuckle print detection using the CHVD and projectile search algorithms for both left and right hands.

where $L_{k, m}$ denotes a set of points that form a line on the lattice $Z_{p}^{2}, k$ defines the slope of the line, and $m$ refers to the coefficients of Ridgelet transform in each direction. These lines are given as

$$
L_{k, m}=\left\{(x, y): y=k x+m \quad(\bmod p) \quad x \in Z_{p}\right\}, 0 \leq k \leq p,
$$

FRIT is obtained by taking the discrete Wavelet transform of each FRAT projection, defined as:

$$
\operatorname{FRIT}[k, n]=\left\langle F R A T[k, m], \omega_{n}^{k}[m]\right\rangle
$$

where $\omega_{n}^{k}$ is the Wavelet basis function of FRAT.

A number of experiments had been carried out by using different wavelet bases and the optimal basis was selected for use. In this research, we adopt Symmlets with one wavelet decomposition level.

\subsubsection{Knuckle Print Representation using Ridgelet Feature}

FRAT can be used to represent knuckle prints which encompass ridge-like patterns. We proposed a blockprocessing scheme to deal with the knuckle print images of different sizes. Every knuckle print image is partitioned into $N$ blocks and FRAT is performed locally on each block. The ridgelet coefficient is then calculated for each of the block to obtain its energy measures, E. Given the ridgelet coefficient as $\varphi_{1, j}, \varphi_{2, j}, \ldots, \varphi_{C_{j}, j}$ of the $j^{\text {th }}$ block, the energy measures are calculated as,

$$
\begin{aligned}
& E(t)=\frac{1}{C_{j}} \sum_{i=1}^{C_{j}}\left|\varphi_{i, j}\right| \\
& E(t+1)=\sqrt{\left(\frac{1}{C_{j}} \sum_{i=1}^{C_{j}} \varphi_{i, j}^{2}\right)}
\end{aligned}
$$

where $t=1,2, \ldots, N$, and $C_{j}$ is the number of ridgelet coefficients in the $j^{\text {th }}$ block. These equations measure the dispersion of the ridgelet coefficients, and normalise the values independent of sub-block size. The energy measures are the knuckle print features. Thus, the feature length for each subject is equals to $N \times 2$ enery measures $\times f$ fingers. The matching scores for two knuckle print features can be computed using Euclidean distance. 


\subsection{Extracting Palm Print Feature}

Even though the line patterns on palm and knuckle prints are similar, we cannot use the same algorithm for the reason that knuckle print contains simpler and horizontal-like line patterns. Therefore, edge-sensitive method such as ridgelet transform would be more suitable. On the other hand, palm print consists of irregular line patterns from different directions and hence another solution is required to extract palm print features. In this paper, we use the Wavelet Gabor Competitive Code (WGCC) technique proposed in our previous work (Michael et al., 2006) as the feature extraction technique for palm print recognition. This technique is chosen because of its robustness and computational economic, making it suitable for real-time applications.

Wavelet Transform is first used to decompose the palm print images into lower resolution representation. After that, 2D Gabor filter is applied on the decomposed images for palm print representation. Figure 5 depicts the palm print features extracted using this approach. Details of the Wavelet Transform and Competitive Gabor code techniques could be found in (Michael et al., 2006). In this research, Hamming distance is deployed as the feature matching tool. It is applied to count the fraction of bits that differ between two competitive code strings.

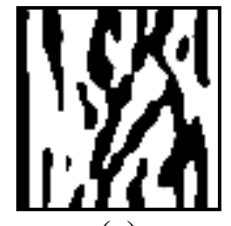

(a)

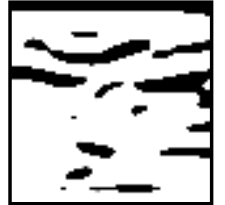

(d)

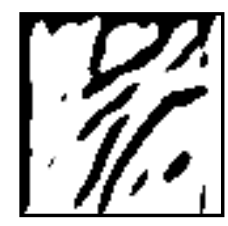

(b)

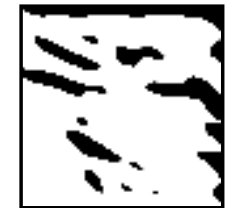

(e)

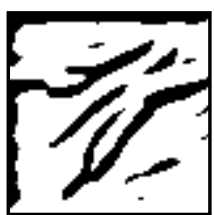

(c)

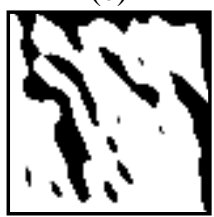

(f)

Figure 5: The palm print textures obtained from 2D Gabor filter with six different angular settings.

\subsection{Fusion Scheme}

In this paper, several decision-level fusion rules are used to combine the palm print and knuckle print matchers. In specific, we have tested our method with AND- and OR-voting rules, sum rule, as well as weighted sum rule.

The AND- and OR-voting rules are the simplest fusion techniques (Chatzis et al., 1999). The AND-voting rule fusion decision is made only when all the classifiers agree. For OR-voting rule fusion, a decision is reached when one of the classifiers makes a decision.

On the other hand, sum rule takes the average of the scores from the two modalities. The summation of both single-modal classifier matching score or distance is calculated as

$$
S=P_{m s}+K_{m s}
$$

where $P_{m s}$ and $K_{m s}$ represent the matching score of palm print and knuckle print respectively and output the class with the smallest value of $S$. The main advantage of this rule is its simplicity, and the fact that it does not need any training.

The last type of fusion scheme adopted in this research is the weighted sum rule. There exist different classifiers with different performances, thus weights can be used to combine the individual classifiers. Since only two models of biometrics are used in our system, the weighted sum $S_{w}$ can be formed as

$$
S_{w}=w P_{m s}+(1-w) K_{m s}
$$

where $w$ is the weight that falls within value from 0 to 1 . The performance of these rules pertaining to our combination will be demonstrated and explained in the following section. 


\section{EXPERIMENT SETUP AND EVALUATION}

The proposed system uses a low-cost web camera mounted on the work area facing upward. In real-time, the system can track the position and orientation of the palm and five fingers of each hand without the use of special markers or gloves, resulting in up to 8 degrees of freedom for each hand.

In this experiment, a standard PC with Intel Pentium 4 HT processor $(3.4 \mathrm{GHz})$ and $1024 \mathrm{MB}$ RAM was used. The program was developed using Visual Studio .NET 2008. We used a 1.3 mega pixel Charge Couple Device (CCD) web camera as the input sensor. The hand image was detected in real-time video sequence at 25 fps. The image resolution was 640 x 480 pixels, with color output type in 256 RGB ( 8 bits-per-channel). The ROIs of the palm print and knuckle print were captured and stored as bitmap format from the video sequence. The delay interval between capturing the current and the next ROI was 2 seconds. The exposure parameter of the web-cam was set to low to reduce the effect of background light as the background light may disrupt the quality of the palm print image. We placed a 9 watt warm-white light bulb beside the camera. The bulb emitted yellowish light source that enhanced the lines and ridges of the palm. A black enclosure made from vinyl plastic was placed around the web-cam and light bulb to set up a semi-controlled environment as shown in Figure 6. The black enclosure can absorb some reflectance from the light bulb so that the palm image will not appear too bright. We also include a dimmer to adjust the brightness of the light bulb.

The proposed methodology was tested on a modest size database containing palm images from 125 individuals. 61 of them are females, 76 of them are less than 30 years old. The users come from different ethnic groups such as Chinese, Malays, Indians, Arabians, Indonesians, Pakistanis, African, Sudanese and Punjabi. Most of them are students and lecturers from our university. The users are requested to stretch their fingers during the image capturing process.

In specific, assume that there are 125 classes represented as $\left\{C_{i} \mid i=1, \ldots, 125\right\}$, and 10 samples in each class denoted as $\left\{\mathrm{C}_{i, j} \mid i=1, \ldots, 125, j=1, \ldots, 10\right\}$. For the genuine test, we compared the images with each other in the same class. For example, $\mathrm{C}_{1,1}$ was compared with $\mathrm{C}_{1,2}, \mathrm{C}_{1,3}, \ldots, \mathrm{C}_{1,10}$ and $\mathrm{C}_{1,2}$ was compared with $\mathrm{C}_{1,3}, \mathrm{C}_{1,4}, \ldots$, $\mathrm{C}_{1,10}$, and so on in class $\mathrm{C}_{1}$. As there were 10 samples in each class, the number of genuine comparisons per class was the $9^{\text {th }}$ triangular number $(45=9+\ldots+2+1)$. Thus, the total number of genuine tests was $5625(45$ comparisons $\mathrm{x} 125$ classes). On the other hand, we compared the sample in one class with the rest of the classes for the imposter test. For instance, $\mathrm{C}_{1,1}$ was compared with $\mathrm{C}_{2,1}, \mathrm{C}_{3,1}, \ldots, \mathrm{C}_{125,1}$, and $\mathrm{C}_{1,2}$ was compared with $\mathrm{C}_{2,2}$, $\mathrm{C}_{3,2}, \ldots, \mathrm{C}_{125,2}$ in class $\mathrm{C}_{1}$, while $\mathrm{C}_{2,1}$ was compared with $\mathrm{C}_{3,1}, \mathrm{C}_{4,1}, \ldots, \mathrm{C}_{125,1}$, and $\mathrm{C}_{2,2}$ was compared with $\mathrm{C}_{3,2}$, $\mathrm{C}_{4,2}, \ldots, \mathrm{C}_{125,2}$ in class $\mathrm{C}_{2}$. Thus the total number of imposter comparison was $78750\left(\left(125^{\text {th }}\right.\right.$ triangular number $=$ 7875 comparisons among all classes) x 10 samples).

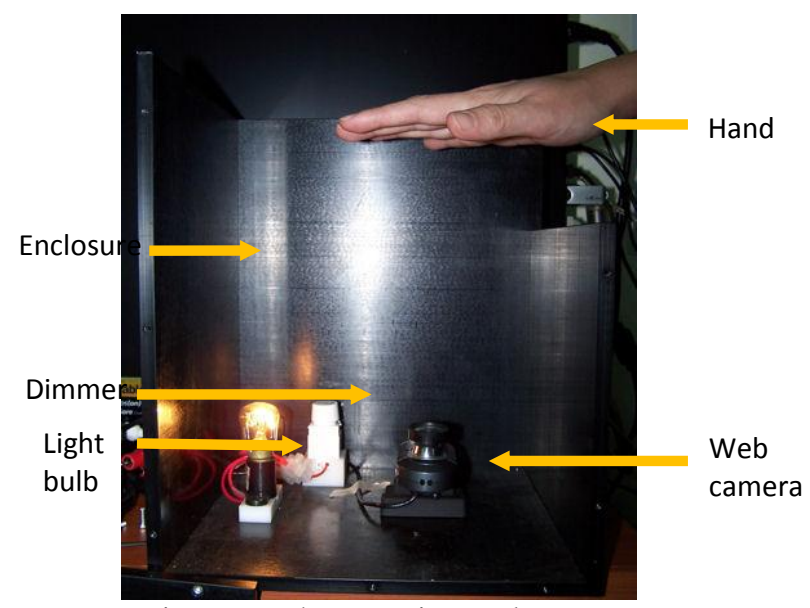

Figure 6: The experimental setup.

\subsection{Biometric Performance Measurements Criterion}

The proposed system can be seen as a two class problems: whether a person should be claimed as a true client or an imposter. In order to evaluate the success of the system, a standard measurement is used to verify the acceptance errors and rejection errors. They are defined as follows:

False Reject Rate (FRR) - the percentage of clients or authorized person that the biometric system fails to accept. It will increase proportionally to the security threshold. When the security threshold increases, more users (including the authorized person) will be rejected due to the high security level. FRR is defined as 


$$
\mathrm{FRR}=\frac{\text { Number of rejected clients }}{\text { Total number of client access }} \times 100 \%
$$

False Acceptance Rate (FAR) - the percentage of imposters or unauthorized person that the biometric system fails to reject. It rises when the security threshold (matching confidence) is lowered. More formally, FAR is defined as

$$
\text { FAR }=\frac{\text { Number of accepted imposter }}{\text { Total number of imposter accesses }} \times 100 \%
$$

Equal Error Rate (EER) - is an optimal rate where FAR is equal to FRR. Graphically, EER is recognized as the crossing point between FAR and FRR. It is commonly used to determine the overall accuracy of the system and serve as comparative measure against the other biometric systems.

These three performance measures, namely FAR, FRR and EER will be used to testify the proposed algorithms in the subsequent sections.

\section{RESULTS AND DISCUSSION}

In this section, we conducted extensive experiments to evaluate the effectiveness and the robustness of the proposed system. We first carried out palm print tracking in a semi-controlled environment to validate the robustness of the proposed palm print and knuckle print tracking techniques. After that, we performed experiments to evaluate the verification performance of the proposed system.

\subsection{Online Palm Print and Knuckle Print Tracking}

The experiment was conducted in the semi-controlled environment shown in Figure 6. A user was asked to put his hand above the web-cam and slowly rotate ( \pm 30 degrees) his hand to the left and right directions. The user was also asked to move his hand closer and gradually away from the web-cam. Some tracking results of the palm print and knuckle print regions are shown in Figure 7. The proposed palm print and knuckle print tracking method performs quite well as the ROIs of the palm print and knuckle print can be located in an average time of 0.026 seconds regardless of changes in size and direction.
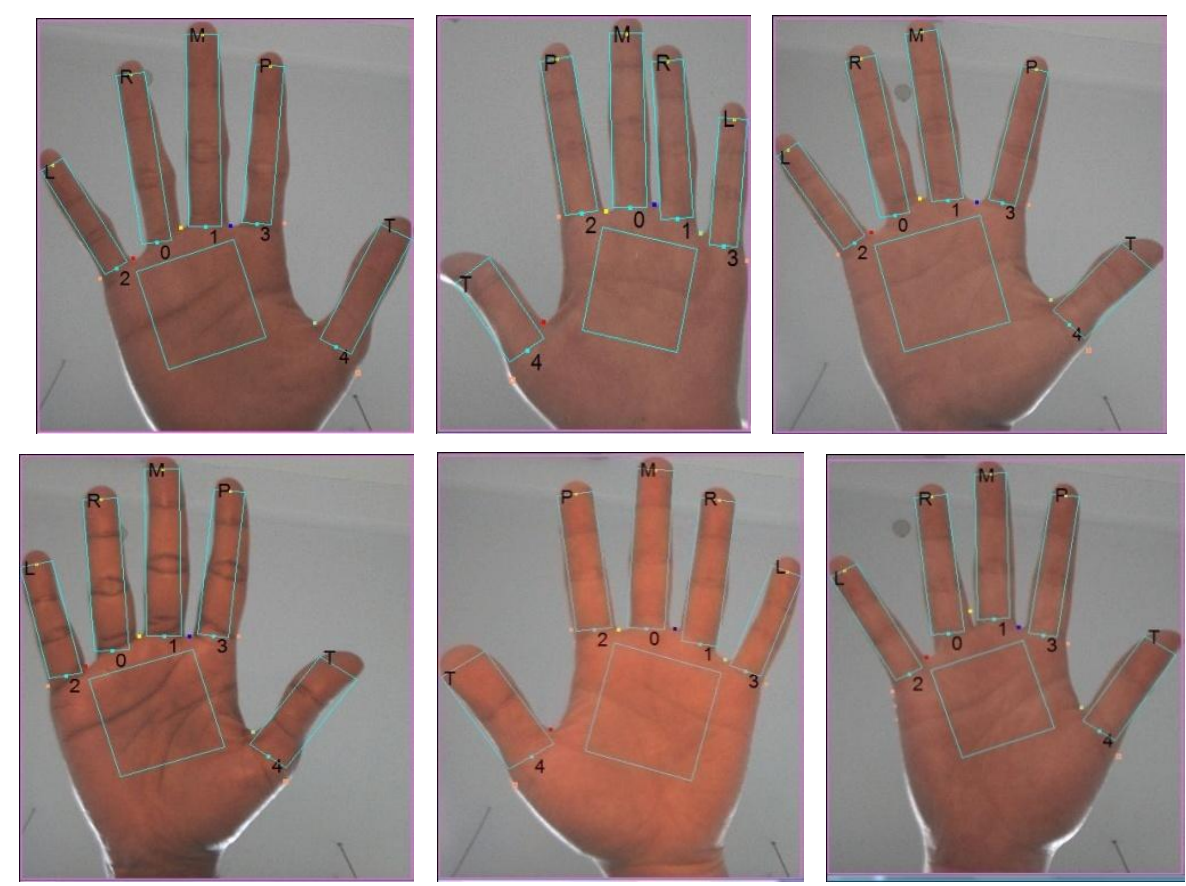

Figure 7: Some of the tracking results of the proposed palm print and knuckle print tracking algorithm. 


\subsection{Knuckle print based verification performance}

An experiment was conducted to evaluate the performance of the knuckle print system. Ridgelet transform was used to extract the knuckle print feature for representation. Table 1 shows the result of the proposed method. It can be observed that ridgelet transform can achieve FAR equals $2.9 \%$ and FRR equals $3.0 \%$, yielding EER of $2.9 \%$. When FAR is set to zero percent, FRR of $12.43 \%$ is obtained. The graph showing the genuine and imposter distribution of this system is illustrated in Figure 8.

Table 1. Knuckle print based verification performance

\begin{tabular}{|c|c|c|c|c|}
\hline Modality & FAR \% & FRR \% & EER \% & $\begin{array}{c}\text { FRR when } \\
\text { FAR }=\mathbf{0 \%}\end{array}$ \\
\hline Knuckle Print & 2.97 & 3.00 & 2.99 & 12.43 \\
\hline
\end{tabular}

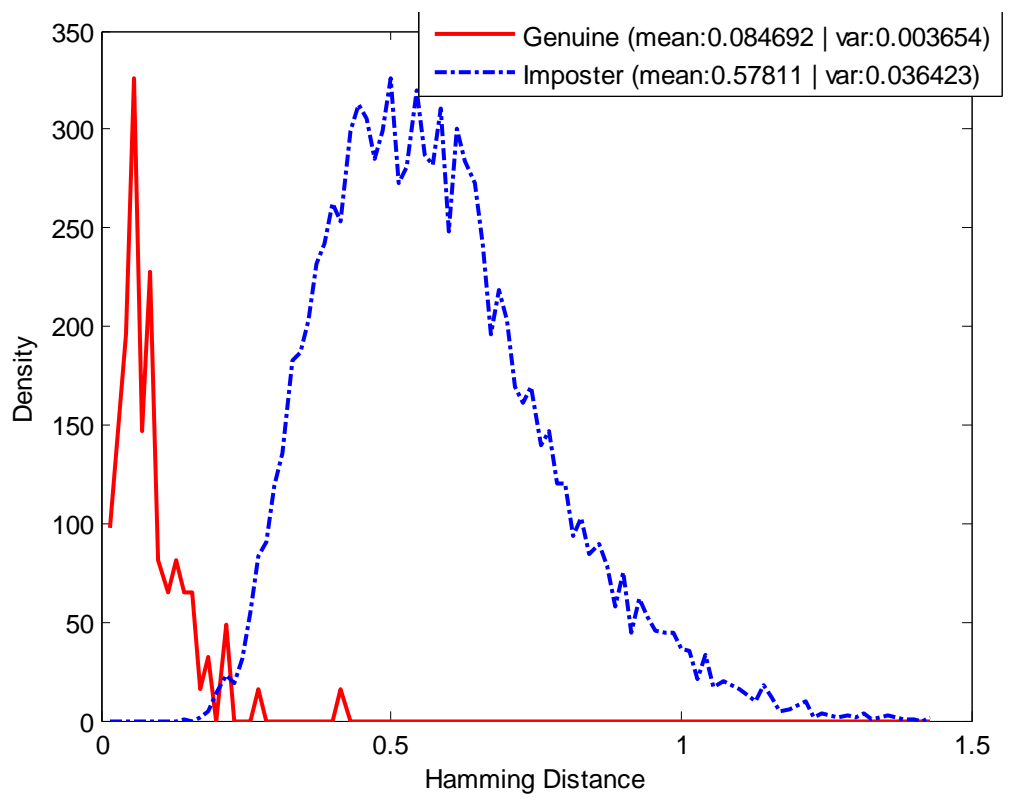

Figure 8: The genuine and imposter distributions of the knuckle print modality based on ridgelet transform.

\subsection{Palm print based verification performance}

The next experiment was carried out to assess the effectiveness of the palm print system. The competitive rule with minimum response was used in this experiment. For WT, we adopted the Symmlet Order 2 wavelet basis with two levels of Wavelet decomposition. The result is displayed in Table 2. The proposed scheme yields FAR $=2.33 \%, \mathrm{FRR}=2.00 \%$, giving rise to $\mathrm{EER}=2.16 \%$ for palm print system. The graph showing the genuine and imposter distributions of the palm print method is illustrated in Figure 9.

Table 2: Palm print based verification performance

\begin{tabular}{|c|c|c|c|c|}
\hline Modality & FAR \% & FRR \% & EER \% & $\begin{array}{c}\text { FRR when } \\
\text { FAR }=\mathbf{0 \%}\end{array}$ \\
\hline Palm Print & 2.33 & 2.00 & 2.16 & 19.25 \\
\hline
\end{tabular}




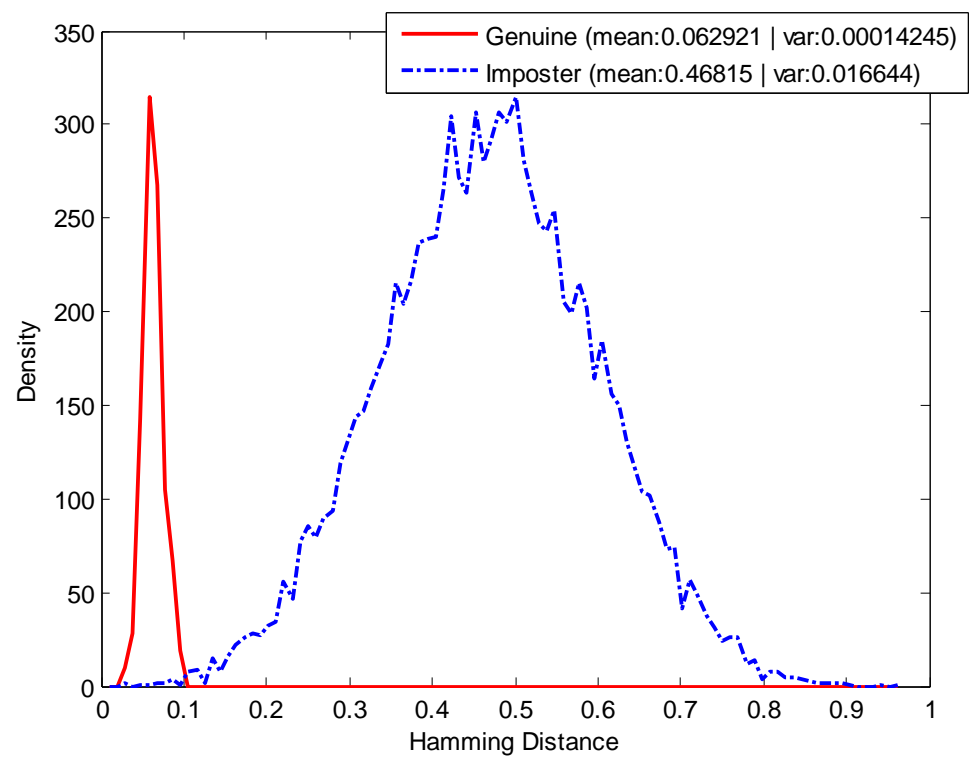

Figure 9: The genuine and imposter distributions of the palm print modality based on competitive rule.

\subsection{Correlation Analysis}

Correlation analysis of individual experts is important to determine their discriminatory power, information complementary ability and data separability. The fusion result can be very effective if the errors made by the classifiers are highly de-correlated (with higher independency). In other words, the higher the de-correlation between the errors made by the classifiers, the more effective the fusion will become. This is due to the reason that more new information will be introduced when the de-correlation between the errors increases (Verlinde, 1999).

One way to visualize the correlation between two classifiers is to plot the distribution graph of the imposter and client populations. In the correlation observation experiment shown in Figure 10, the distribution of the imposter and client populations take the form of two nearly independent clusters. This indicates that the correlation between the individual palm print and knuckle print classifier is very low.

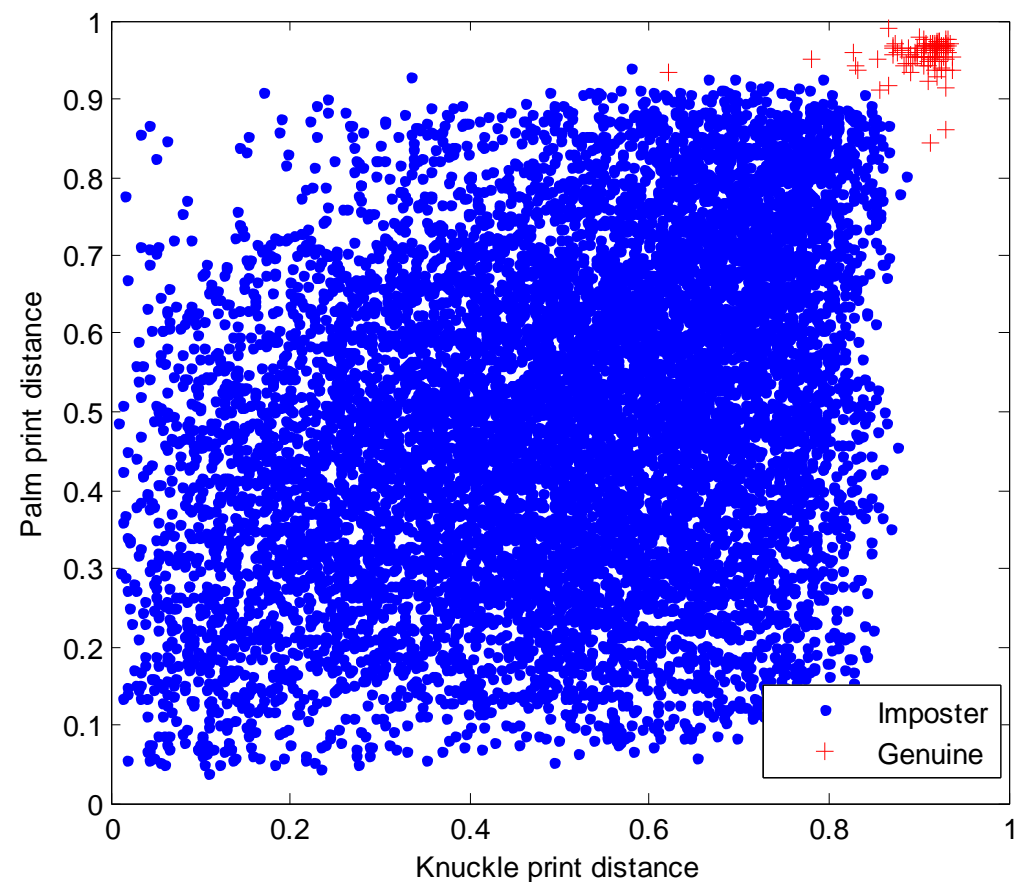

Figure 10: Visual representation of the correlation of between two individual palm print and knuckle print classifiers. 
Table 3: Correlation matrices of hand geometry and palm print decision scores

\begin{tabular}{|c|c|c|}
\hline Correlation & Knuckle Print & Palm Print \\
\hline Knuckle Print & 1.000 & 0.002 \\
\hline Palm Print & 0.002 & 1.000 \\
\hline
\end{tabular}

The result of the correlation matrices is shown in the Table 3. It can be observed that the correlation between the palm print and knuckle print are very small. This implies that both biometrics are highly independent and are suitable to be used for fusion.

\subsection{Verification Performance of Fusion Approach}

As described in Section 2.4, different fusions approaches are used in this research to combine the decision scores produced by the knuckle print and palm print experts. The decision scores are normalized to range of [0, 1] before entering the fusion module. This is to avoid attributes in greater numeric ranges to dominate those in smaller numeric ranges and deflect numerical difficulties during the calculation.

For AND- and OR-voting rules, we need to choose an optimal threshold value from both palm print and knuckle print experts. Based on our previous experiment, we choose the threshold value based on the EER\% performance. Thus, the palm print expert threshold value is set to 0.163 (after normalization). On the other hand, the threshold for knuckle print expert is set to 0.24 . The sum rule fusion was performed by straight forward addition of the decision vectors as shown in Equation 4. The weighted sum rule fusion weight setting was found via iterative procedure by decrementing the value of $w$ from 1 to 0 according to Equation 5. The results of the fusion are presented systematically in Table 4.

Table 4: Result of applying the fusion rules

\begin{tabular}{|c|c|c|c|c|}
\hline Fusion Scheme & FAR \% & FRR \% & EER \% & FRR when FAR = 0\% \\
\hline AND rule & 1.74 & 2.00 & 1.87 & 10.02 \\
\hline OR rule & 1.52 & 1.52 & 1.52 & 8.47 \\
\hline Sum rule & 1.61 & 1.59 & 1.60 & 8.89 \\
\hline $\begin{array}{c}\text { Weighted sum } \\
\text { rule }\end{array}$ & 1.25 & 1.25 & 1.25 & 5.94 \\
\hline
\end{tabular}

It can be observed that all the fusion schemes perform better than the individual classifiers. As for the result obtained by using OR rule, the performance is better than that of AND rule. On the other hand, the result of sum rule is poorer than the OR-voting rule as there is no tuning parameter to fine tune the sum rule. Among the fusion schemes, weighted sum rule with optimum setting of $w=0.7$ gives the best performance result. Table 4 shows dramatic decrement of EER for the fusion methods. For the case where FAR $=0 \%$ is selected to obverse the FRR behaviour. We observed that all fusion schemes are able to reduce the FRR compare to each individual classifier. However, weighted sum rule is able to maintain the low FRR as obtained in Table 4, while other combined classifiers and individual classifiers suffer from the higher FRR.

\section{CONCLUSIONS}

This paper presents a low resolution contact-less palm print and knuckle print recognition system. The proposed contact-less palm print recognition system offers several advantages like flexibility and user-friendliness. We proposed a novel hand tracking algorithm to automatically detect and locate the ROIs of the palm print and knuckle print. The proposed algorithm works well under semi-controlled environment. Extensive experiments have been conducted to evaluate the performance of the system in both on-line and off-line settings. Our approach produces promising result of EER as low as $1.25 \%$ and FRR is $5.94 \%$ when FAR $=0 \%$ by using weighted sum rule as our fusion scheme. 
Although the proposed system works satisfactory in semi-controlled environment, further investigation should be conducted to verify its effectiveness under other types of open-environments. Besides, more users should be included into the database to test its feasibility to be used in medium to large organizations. In future, we will conduct more rigorous tests to validate the performance of the recognition system in real-time applications.

\section{REFERENCES}

Hand-based Biometrics, Biometric Technology Today, Vol 11, No. 7, pp. 9-11, 2003.

W. Boles, and S. Chu, "Personal identification using images of the human palms", Proceedings of IEEE Region 10 Annual Conference, Speech and Image Technologies for Computing and Telecommunications, Vol. 1, pp. 295-298, 1997.

E.J. Candes, D.L. Donoho, "Ridgelets: a key to higher-dimensional intermittency," Phil. Trans. Roy. Soc. Lond., Vol. 357, pp. 2495-2509, 1999.

V. Chatzis, A.G. Bors, I. Pitas, "Multimodal decision-level fusion for person authentication," IEEE Trans. on Systems, Man and Cybernetics, Part A: Systems and Humans, Vol 29(6), pp. 674-681, 1999.

J. Chen, C. Zhang, G. Rong, "Palmprint recognition using creases", Proceedings of International Conference of Image Processing, pp. 234-237, 2001.

T. Connie, A. Jin, M. Ong, D. Ling, “An automated palmprint recognition system”, Image and Vision Computing, Vol. 23, No. 5, pp.501-515, 2005.

S.R. Deans, “The Radon Transform and Some of Its Applications,” New York: Wiley, 1983.

M.N. Do and M. Vetterli, "The finite ridgelet transform for image representation,” IEEE Trans. Image Process., Vol 12(1), pp. 16-28, 2003.

D.L. Donoho, “Orthonormal ridgelets and linear singularities,” SIAM J. Math. Anal., Vol 31(5), pp. 1062-1099, 2000.

N. Duta, A. Jain, K. Mardia, "Matching of palmprint”, Pattern Recognition Letters, Vol. 23, pp. 477-485, 2002.

J. Funada, N. Ohta, M. Mizoguchi, T. Temma, T. Nakanishi, K. Murai, "Feature extraction method for palmprint considering elimination of creases", Proceedings of the 14th International Conference of Pattern Recognition, pp. 1849-1854, 1998.

D. Huang, W. Jia, D. Zhang, "Palmprint verification based on principal lines", Pattern Recognition, Vol. 41, No. 4, pp. 1316-1328, 2008.

S. Kung, S. Lin, M. Fang, “A neural network approach to face/palm recognition”, Proceedings of IEEE Workshop on Neural Networks for Signal Processing, pp. 323-332, 1995.

M. Leung, A. Fong, H. Cheung, "Palmprint verification for controlling access to shared computing resources", IEEE Pervasive Computing, Vol. 6, No. 4, pp. 40-47, 2007.

W. Li, D. Zhang, Z. Xu, "Palmprint Identification by Fourier Transform," International Journal of Pattern Recognition and Artificial Intelligence, Vol. 16(4), pp. 417-432, 2003.

Q. Li, Z. Qiu, D. Sun, J. Wu, "Personal Identification using knuckleprint,” SINOBIOMETRICS, Guangzhou, Vol 2004, pp. 680-689, December 2004.

N. Loris, L. Alessandra, "A multi-matcher system based on knuckle-based features," Neural Computing and Applications, Springer London, Vol 18, No. 1, pp. 87-91, 2009. 
G. Lu, D. Zhang, K. Wang, "Palmprint recognition using eigenpalms features," Pattern Recognition Letters, Vol. 24, Issues 9-10, pp. 1473-1477, 2003.

G. Michael, T. Connie, T. Andrew, N. David, “A Fast Palm Print Verification System,” 3rd International Conference on Computer Graphics, Imaging and Visualization, pp. 168-172, 2006.

G. Michael, T. Connie, T. Andrew, "Touch-less palm print biometrics: Novel design and implementation," Image and Vision Computing, Vol 26, Issue 12, pp. 1551-1560, December 2008.

M. RafaelDiaz, C. Travieso, J. Alonso, M. Ferrer, "Biometric system based in thefeature of hand palm", Proceedings of 38th Annual International Carnahan Conference on Security Technology, pp. 136-139, 2004.

S. Ribaric, I. Fratric, “A biometric identification system based on eigenpalm and eigenfinger features," IEEE Trans Pattern And Machine Intelligence, Vol. 27(11), pp. 1698-1709, 2005.

P. Verlinde, “A Contribution to Multi-Modal Identity Verification Using Decision Fusion”, PhD Thesis, Department of Signal and Image Processing, Telecom Paris, France, 1999.

X. Wu, K. Wang, D. Zhang, "Wavelet based palmprint recognition", Proceedings of the First International Conference on Machine Learning and Cybernetics, Vol. 3, pp. 1253-1257, 2002.

X. Wu, D. Zhang, K. Wang, "Fisherpalms based palmprint recognition," Pattern Recognition Letter, Vol 24, pp. 2829-2838, 2003.

X. Wu, K. Wang, D. Zhang, “A novel approach of palm-line extraction”, Proceeding of the Third International Conference on Image and Graphics, pp. 230-233, 2004.

J. You, W. Kong, D. Zhang, K. Cheung, “On hierarchical palmprint coding with multiple features for personal identification in large databases", IEEE Transactions on Circuits and Systems for Video Technology, Vol. 14, No. 2, pp. 234-243, 2004. 